International Journal of Pure and Applied Mathematics

Volume $97 \quad$ No. 2 2014, 177-181

ISSN: 1311-8080 (printed version); ISSN: 1314-3395 (on-line version)

url: http://www.ijpam.eu

doi: http://dx.doi.org/10.12732/ijpam.v97i2.5

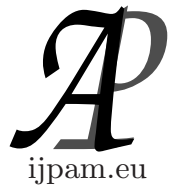

\title{
THE SOLUTION OF FERMAT EQUATION IN THE RATIONAL POINTS OF THE UNITARY CIRCUMFERENCE
}

\author{
B. Martin Cerna Maguiña \\ Department of Mathematics \\ National University of "Santiago Antúnez de Mayolo" \\ Campus Shancayan, Av. Centenario 200, Huaraz, PERÚ
}

\begin{abstract}
In this work we resolve the Fermat equation over rational points in the unitary circumference. For this we take a point $\left(p_{0}, q_{0}\right)$ in the unitary circumference $x^{2}+y^{2}=1$, where $p_{0}, q_{0} \in \mathbb{Q}$ or $q_{0} \in \mathbb{I}$ and $p_{0} \in \mathbb{I}$. Then the straight line $y=q_{0}$ intersects the curve $x^{d}+y^{d}=1$, in the point $\left(p_{1}, q_{0}\right)$ we demonstrated that $p_{1}$ is an irrational number.
\end{abstract}

AMS Subject Classification: 11A41, 11D41

Key Words: primes, Fermat's equations, Diophantine equations

\section{Introduction}

We know that Fermat's equation is $a^{n}+b^{n}=c^{n}$, this doesn't have different integer solution of the trivial for $a, b, c, n \in \mathbb{Z}^{+}$and $n \geq 3$, it was solved for the English Mathematician Andrew Wile in 1996; I listened that this only was possible using a technique of extremely difficult for its comprehension. Motivated by these comments, I took the decision investigate, so I discovered a simple technique that can help solve the general case.

Received: May 8, 2014

(c) 2014 Academic Publications, Ltd. url: www.acadpubl.eu 
The used technique, consist in the following, We took a point in the Cartesian plane whose coordinates are $\left(p_{0}, q_{0}\right)$ in the unitary circumference, where $p_{0}, q_{0} \in \mathbb{Q}$ or $q_{0} \in \mathbb{Q}$ and $p_{0} \in \mathbb{I}$, then we get $p_{0}^{2}+q_{0}^{2}=1$, these points are known as

$$
p_{0}=\frac{2 t_{0}}{1+t_{0}^{2}}, \quad q_{0}=\frac{1-t_{0}^{2}}{1+t_{0}^{2}},
$$

here note that $p_{0}, q_{0} \in \mathbb{Q}^{+}$if and only if $t_{0} \in \mathbb{Q}^{+}, t_{0} \in[0,1]$, if $q_{0} \in \mathbb{Q}^{+}$and $p_{0} \in \mathbb{I}^{+}$then $t_{0}^{2} \in \mathbb{Q}^{+}$.

The straight line $y=q_{0}$, in the first quadrant intersects the curve $x^{d}+y^{d}=1$ in the point $\left(p_{1}, q_{0}\right)$, then we have the following equation $p_{1}^{d}+q_{0}^{d}=1$.

From the last equation we demonstrate that $p_{1}$ never is a rational number. So it is important to note that $\left(p_{0}, q_{0}\right)$ also can be written as:

$$
q_{0}=\frac{2 t_{1}}{1+t_{1}^{2}}, \quad p_{0}=\frac{1-t_{1}^{2}}{1+t_{1}^{2}}
$$

where the relation between $t_{0}, t_{1}$ is $t_{0}=\frac{1-t_{1}}{1+t_{1}}$.

Let us introduce the notations used in this work: $\mathbb{Z}^{+}$represents the set of positive integer numbers, $\mathbb{Q}$ represents the set rational numbers, $\mathbb{I}$ represents the set of irrational numbers.

\section{Fermat Had Reason}

Theorem 1. Let $a, b, c, d \in \mathbb{Z}^{+}$the equation $a^{d}+b^{d}=c^{d}$ does not have integer solution different of the trivial.

Proof. This theorem is equivalent to prove the following $p^{d}+q^{d}=1$ it does not have rational solution para $p, q \in \mathbb{Q} \cap[0,1]$. So we take $\left(p_{0}, q_{0}\right)$ a point in the unitary circumference. Then following cases happens:

I) $p_{0}, q_{0} \in \mathbb{Q}$ and satisfies the equation $p_{0}^{2}+q_{0}^{2}=1$, where

$$
p_{0}=\frac{2 t_{0}}{1+t_{0}^{2}}=\frac{1-t_{1}^{2}}{1+t_{1}^{2}} \text { and } q_{0}=\frac{1-t_{0}^{2}}{1+t_{0}^{2}}=\frac{2 t_{1}}{1+t_{1}^{2}}
$$

Clearly $t_{0}, t_{1} \in \mathbb{Q}$ and $t_{0}, t_{1}$ are related by the following equation

$$
t_{0}=\frac{1-t_{1}}{1+t_{1}}
$$


Let $t_{0}=\frac{m}{n}, t_{1}=\frac{m_{1}}{n_{1}}$, where $m, n, m_{1}, n_{1} \in \mathbb{Z}^{+}$and $m, n$ are relative primes.

The same way $m_{1}, n_{1}$ are relative primes and satisfies the relation $m<n$, $m_{1}<n_{1}$.

From (2) we have

$$
t_{0}=\frac{m}{n}=\frac{n_{1}-m_{1}}{n_{1}+m_{1}}
$$

The line $y=q_{0}$ intersects the curve $x^{d}+y^{d}=1$ in the point $\left(p_{1}, q_{0}\right)$, therefore this point satisfies the following :

$$
p_{1}^{d}+q_{0}^{d}=1
$$

From the relation (1),(3) and (4) we have:

$$
p_{1}=\frac{\sqrt[d]{\left(m^{2}+n^{2}\right)^{d}-\left(n^{2}-m^{2}\right)^{d}}}{n^{2}+m^{2}}=\frac{\sqrt[d]{\left(n_{1}^{2}+m_{1}^{2}\right)^{d}-\left(2 n_{1} m_{1}\right)^{d}}}{n_{1}^{2}+m_{1}^{2}}
$$

Supposing that $p_{1} \in \mathbb{Q}$ from the relation (4) and (5) we have the equation

$$
A^{d}+\left(2 n_{1} m_{1}\right)^{d}=\left(n_{1}^{2}+m_{1}^{2}\right)^{d}
$$

If $A=n_{1}^{2}+m_{1}^{2}-j, j=1,2, \ldots, n_{1}^{2}+m_{1}^{2}-1$, we have

$$
\left(n_{1}^{2}+m_{1}^{2}-j\right)^{d}+\left(2 n_{1} m_{1}\right)^{d}=\left(n_{1}^{2}+m_{1}^{2}\right)^{d}
$$

don't have a solution, for all $n_{1}, m_{1}, d \in \mathbb{N}$ and $\forall j$ odd number.

Therefore

$$
\left(n_{1}^{2}+m_{1}^{2}-j\right)^{d}+\left(2 n_{1} m_{1}\right)^{d}>\left(n_{1}^{2}+m_{1}^{2}\right)^{d}
$$

or

$$
\left(n_{1}^{2}+m_{1}^{2}-j\right)^{d}+\left(2 n_{1} m_{1}\right)^{d}<\left(n_{1}^{2}+m_{1}^{2}\right)^{d}
$$

Then We supposing that exists $j_{0}$ even number such that (6) have a solution

$$
\left(n_{1}^{2}+m_{1}^{2}-j_{0}\right)^{d}+\left(2 n_{1} m_{1}\right)^{d}=\left(n_{1}^{2}+m_{1}^{2}\right)^{d}
$$

From the relation (9) and (7) we have

$$
\left(n_{1}^{2}+m_{1}^{2}-j\right)^{d}>\left(n_{1}^{2}+m_{1}^{2}-j_{0}\right)^{d},
$$

if $d$ is an odd number we have

$$
-j>-j_{0} \Rightarrow j_{0}>j, \forall j \text { odd number. }
$$


Then $j_{0}=n_{1}^{2}+m_{1}^{2}-1$, if $n_{1}$ is an odd number and $m_{1}$ is an even number or viceversa. This relation and (9) is

$$
1^{d}+\left(2 n_{1} m_{1}\right)^{d}=\left(n_{1}^{2}+m_{1}^{2}\right)^{d}
$$

which don't have an integer solution.

From the relation (8) and (9), we have

$$
\left(n_{1}^{2}+m_{1}^{2}-j\right)^{d}<\left(n_{1}^{2}+m_{1}^{2}-j_{0}\right)^{d},
$$

therefore, if $d$ is an odd number

$$
j_{0}<j, \forall j \text { odd number, }
$$

for $j=1$, we have that $j_{0}$ doesn't exist.

When $d$ is a even number the analysis is the same

II) If $q_{0} \in \mathbb{Q}$ and $p_{0} \in \mathbb{I}$. We have

$$
p_{0}^{d}+(n-m)^{d}=(n+m)^{d}
$$

for $B=n+m-j, j=1,2, \ldots, n+m-1$, we have

$$
(n+m-j)^{d}+(n-m)^{d}=(n+m)^{d},
$$

for $j=n+m-k$ on (10), we have

$$
k^{d}+(n-m)^{d}=(n+m)^{d}
$$

If $k$ is an odd number, then (11) don't have a solution for every $n, m \in \mathbb{N}$ and $\forall k$ odd number. by analogy with the item (I), We have that (11) only has a solution if

$$
(n+m-1)^{d}+(n-m)^{d}=(n+m)^{d}
$$

where $n$ is odd and $m$ is even number.

Theorem 2. For $d=4$ the equation (12) doesn't solution 
Proof. Suppose that (12) have a solution

$$
\begin{gathered}
(n+m-1)^{4}=(n+m)^{4}-(n-m)^{4} \\
(n+m-1)^{4}=8 n m\left(n^{2}+m^{2}\right) ; n \text { is odd and } m \text { is even }
\end{gathered}
$$

We have $n^{2}+m^{2}=c^{2}, c \in \mathbb{N}$ then

$$
n=u^{2}-v^{2}, m=2 u v, c=u^{2}+v^{2}
$$

Therefore $u$ and $v$ have a square root exact. Furthermore $n$ has a square root exact and $n=(u-v)(u+v)$ then $u=A^{2}, v=B^{2} ; A, B \in \mathbb{N}$. Hence

$$
\left\{\begin{array}{l}
A^{2}-B^{2}=\tau^{2}, \tau \in \mathbb{N} \\
A^{2}+B^{2}=\lambda^{2}, \lambda \in \mathbb{N}
\end{array}\right.
$$

Then (15) don't have an integer solution

\section{Acknowledgments}

The author thanks Victor Pocoy Y. for the reading the manuscript.

\section{References}

[1] Pierre Samuel, Thorie Algbrique des Nombres, Hermann Editeurs Des Sciences Et Des Arts. Francia (1921). 
